\title{
Correction: Current Studies and Applications of Shuffled Frog Leaping Algorithm: A Review
}

\author{
Bestan B. Maaroof ${ }^{1} \cdot$ Tarik A. Rashid $^{2}$. Jaza M. Abdulla ${ }^{3,4} \cdot$ Bryar A. Hassan $^{5}$ (D) Abeer Alsadoon $^{5,6,7}$. \\ Mokhtar Mohammadi $^{8} \cdot$ Mohammad Khishe $^{9} \cdot$ Seyedali Mirjalili ${ }^{10,11}$
}

๑) The Author(s) under exclusive licence to International Center for Numerical Methods in Engineering (CIMNE) 2022

\section{Correction to: \\ Archives of Computational Methods in Engineering https://doi.org/10.1007/s11831-021-09707-2}

This correction is published as Fig. 2 was incorrectly reproduced and has been now updated.

Original article has been now corrected.

Publisher's Note Springer Nature remains neutral with regard to jurisdictional claims in published maps and institutional affiliations.

The original article can be found online at https://doi.org/10.1007/ s11831-021-09707-2.

\section{Bryar A. Hassan}

bryar.hassan@kissr.edu.krd

Tarik A. Rashid

tarik.ahmed@ukh.edu.krd

1 Information Technology Department, College of Commerce, University of Sulaimani, Sulaymaniyah, KRI, Iraq

2 Computer Science and Engineering, University of Kurdistan Hewler, Erbil, KRI, Iraq

3 Computer Science Department, College of Science, Komar University of Science and Technology, Sulaymaniyah, KRI, Iraq

4 Information Technology, College of Commerce, University of Sulaimani, Sulaymaniyah, KRI, Iraq

5 Kurdistan Institution for Strategic Studies and Scientific Research, Sulaimani, Iraq
6 School of Computing and Mathematics, Charles Sturt University, Sydney, Australia

7 Information Technology Department, Asia Pacific International College (APIC), Sydney, Australia

8 Department of Information Technology, College of Engineering and Computer Science, Lebanese French University, Kurdistan Region, Iraq

9 Department of Marine Electronics and Communication Engineering, Imam Khomeini Marine Science University, Nowshahr, Iran

10 Centre for Artificial Intelligence Research and Optimization, Torrens University, Adelaide, Australia

11 Yonsei Frontier Lab, Yonsei University, Seoul, Korea 